\title{
A Comparative Study on Use of Manual Versus Digital Method using Mobile Application for Cephalometric Measurements
}

\author{
Dr Raju Shrestha,' Dr Santosh Kandel ${ }^{2}$ \\ Lecturer, Department of Dentistry, Orthodontic Unit \\ Lumbini Medical College, Pravas, Palpa
}

Correspondence: Dr Raju Shrestha; Email: drrajushrestha1989@gmail.com

\section{ABSTRACT}

Introduction: Various computer software and smartphone applications have been developed for digital cephalometric analysis with ease and in short time. The objective of this study was to compare linear and angular cephalometric measurements obtained, and cephalometric tracing duration between smartphone application tracing (app method) and conventional tracing (manual method) methods.

Materials \& Method: 35 digital Lateral cephalometric radiographs of patient who came for orthodontic treatment were collected. Measurements of cephalometric parameters of composite analysis were obtained with manual and smartphone application (OneCeph) method and compared statistically. Cephalometric tracing duration was also compared between two methods.

Result: Cephalometric tracing duration using manual method was found to be significantly higher $(p<.001)$ than that of the app method. There was no significant difference in values of SNA, SNB, ANB, Nasion perpendicular to point A, Nasion perpendicular to pogonion (pog), Angle of convexity, Go-GN/SN, FMA, U1-NA (degree), U1-NA (linear), L1-NB (degree), Cant of Occlusion, Interincisal angle, Nasolabial angle, Upper lip to Steiner's $S$ line, Lower lip to Steiner's $S$ line obtained with the manual and application method. The values of linear measurement between Lower incisor to NB line showed significant difference $(p<0.05)$.

Conclusion: Although there was no significant difference in cephalometric measurement, cephalometric tracing duration of cephalometric analysis using Smartphone application was significantly faster than that of conventional method.

Keywords: Cephalometric analysis, Cephalometric tracing, Digital method, Manual method, OneCeph, Smartphone application.

\section{INTRODUCTION}

In 1931, Broadbent in USA and Hofrath in Germany simultaneously presented a standardized cephalometric technique using a high powered X-ray machine and a head holder called a Cephalostat or cephalometer.' Cephalometric analysis has been widely used since then in field of orthodontics for case diagnosis, treatment planning, evaluation of treatment progress, evaluation of treatment results, and prediction of growth. Cephalometric analysis can be performed with manual or digital method. Traditional cephalometric analysis is performed by identifying radiographic landmarks on acetate transparent sheet and measuring the linear and angular values with a protractor and ruler. The advances in the field of computer science have led to the widespread use of computers in orthodontic cephalometry. ${ }^{2}$ The Dolphin imaging software (Dolphin Imaging and Management Solutions, Chatsworth, CA), was the first digital innovation that was employed in the orthodontic field which was introduced in $1994 .{ }^{3}$ Manual approach is the oldest and most widely used. ${ }^{4}$

Mobile technologies nowadays have undoubtedly become like a compulsion in daily life. According to study by Millward Brown in 2014, conducted in 30 countries around the world, mobile devices account for $47 \%$ of the total daily screen time. ${ }^{5}$ Smartphone technologies also has a widespread use for various purposes on the medical field. In daily medical practice, smartphone play important roles that range from patient monitoring and diagnostics to effective medical education and communication. ${ }^{6}$ With various smartphone applications and software available which are easy to use and fast, the associated 
technologies are being adopted by clinicians, medical researchers, medical students, patients and health care organizations..$^{6,7}$

Use of traditional manual cephalometric analysis method requires longer time and has chances of error in identifying landmarks, and measuring angle and distance. ${ }^{3}$ In computer-assisted cephalometric analysis, angles and distances are automatically calculated that can eliminate errors in drawing lines between landmarks and in measurements with a protractor. ${ }^{2}$ Besides computer-assisted cephalometric analysis software, smartphone application to carry out various cephalometric analysis has been developed which are fast and simple to use. At present cephalometric analysis software like OneCeph in the application form can be downloaded on smart-phones that can be used to carry out various cephalometric analyses. OneCeph app is reliable, user-friendly which facilitates its use by the clinician on a regular basis. ${ }^{8}$

The objective of this study was to compare linear and angular cephalometric measurements obtained with smartphone application tracing (app method) and conventional tracing (manual method) methods. An additional goal of this study was to evaluate the difference in cephalometric tracing duration between the application method and conventional manual method. Another goal was to assess the reliability of the smartphone-based approach as a cephalometric tracing method for orthodontics. The alternate hypothesis was that the results of various cephalometric measurement performed using a smartphone application would be significantly different from those obtained from measurements performed by hand.

\section{MATERIALS AND METHOD}

Minimum sample size for the study was 25 obtained using sample size calculation formula for comparing two means (paired/ before -After) where alpha=0.05, Beta $=0.2$ mean of difference $=1.263$, standard deviation of difference $=1.505$. Mean of difference and standard deviation of difference was obtained for similar previous study of Sayar et al.8 This cross-sectional study consists of 35 digital Lateral cephalometric radiographs of patients who came for orthodontic treatment were collected from Department of dental surgery: orthodontic unit, Lumbini medical college. The lateral cephalometric radiographs were obtained with patient in Natural Head Position. The inclusion criteria were: 1) Cephalogram of good quality with all landmarks clearly visible, 2) Presence of central incisors and first molars on cephalogram, 3) Cephalogram with teeth in maximum intercuspation. Single examiner (XXX) performed identification of landmarks and all the cephalometric measurement using both the manual and application tracings to minimize the variability of the measurements. The Institutional review Committee of Lumbini Medical College approved this study under Protocol Number IRC-LMC 04-A 1019.Informed consent was taken from the patient.

\section{Manual cephalometric tracing method}

Transparent tracing paper (Straight Line Acetate Tracing Paper) with dimensions of $8 \times 10$ inches and a thickness of $0.003 \mathrm{~mm}$ was placed on the hard copies of lateral cephalograms. Calibration was done before printing the images. Tracing was performed using a $3 \mathrm{Hmechanicalpencil} \mathrm{with} \mathrm{a} 0.3-\mathrm{mm}$ lead tip and using illuminated radiographic viewing screen. Composite analysis involving eight skeletal measurements, five dental measurements and 3 soft tissue measurements were performed. All the landmarks were traced and after that all the lines and planes to be used in the analysis were obtained. The angular and linear hard and soft tissue parameters used in the study are listed below.

\section{Skeletal measurements}

- SNA: Angle between sella nasion and point A. (Steiner's Analysis)

- SNB: Angle between sella nasion and point B. (Steiner's Analysis)

- $\quad$ ANB: Difference between SNA and SNB. (Steiner's Analysis)

- Nasion perpendicular to point A. (McNamara's analysis)

- Nasion perpendicular to pogonion (Pog). (McNamara's analysis)

- Angle of convexity. (Down's Analysis)

- Go-GN/SN. (Steiner's Analysis)

- FMA. (Tweed analysis)

\section{Dental measurements}

U1-NA (degree): Angle between upper incisors to NA line. (Steiner's Analysis) 
- $\quad$ L1-NB (degree): Angle between lower incisors to NB line. (Steiner's Analysis)

- Cant of Occlusion (Down's Analysis) Interincisal angle (Steiner's analysis)

- Ul-NA (mm): Linear distance between labial surfaces of upper incisor to NA line. (Steiner's analysis)

- L1-NB (mm): Linear distance between labial surfaces of lower incisor to NB line. (Steiner's analysis)

\section{Soft Tissue measurements}

- Upper lip protrusion: upper lip to Steiner's S line. (Steiner's analysis)

- Lower lip protrusion: lower lip to Steiner's S line. (Steiner's analysis)

Nasolabial Angle. (Mcnamars's analysis)

All the angular and the linear measurement were performed with a cephalometric protractor and ruler and the data were tabulated for statistical analysis. Also the time required for completing cephalometric measurement of each lateral cephalogram was evaluated using stop watch and the time results were tabulated for statistical analysis.

\section{Smartphone app cephalometric tracing method}

After manual tracing, application tracings of the same lateral cephalograms were performed. Cephalometric analysis using both methods was carried out over 6 days to prevent examiner fatigue. A smartphone
(Samsung galaxy note 3 with android software) was used to perform cephalometric analysis using mobile application. Mobile application known as OneCeph version beta 7 developed by NXS, designed by Dr.M. Pavan Kumar was downloaded for the Google play store (Figure 1). The 35 digital lateral cephalogram radiographs were photographed with mobile camera and saved in a separate folder in photo gallery. All the images were given a separate identification number. Image of cephalogram to be analysed was chosen from the photo gallery in OneCeph app, then the known distance was marked on the cephalogram using the scale in the cephalogram for calibration purpose (Figure 2). After that among various option of different cephalometric analysis, favorites in the toolbar were chosen. In favorites all the cephalometric parameters above mentioned was selected from various analysis like Steiner's analysis, Down's analysis, Holdaway, Jarabak's, McNamara and Tweed. Next all the necessary landmarks were marked with S pen which is integrated stylus pen available Samsung note series phones and measurements were performed (Figure 3 , 4). After that all the cephalometric parameters were measured and tabulated for statistical analysis. Cephalometric tracing duration was also tabulated for statistical analysis.

In order to evaluate the intra examiner error and reliability, 10 randomly selected cephalogram were retraced by same investigator at the interval of 10 days and Interclass correlation coefficients (ICCs) were calculated for each parameter

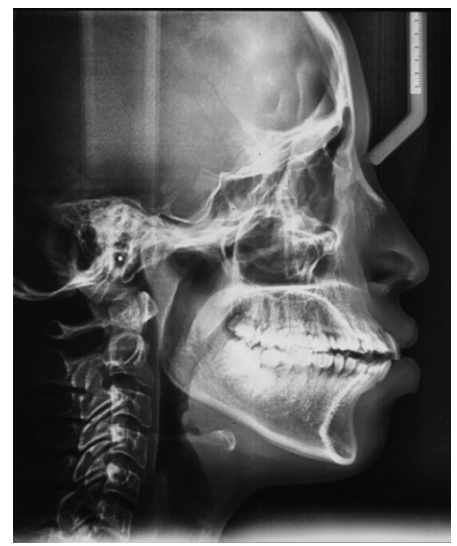

Figure 1: Image of cephalogram Selected in one ceph

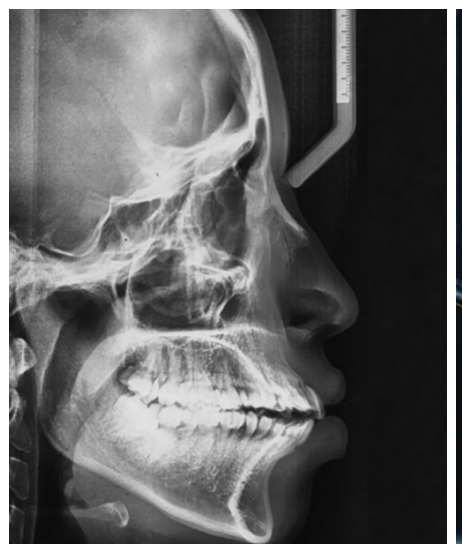

Figure 2: Marking the known distance for calibration

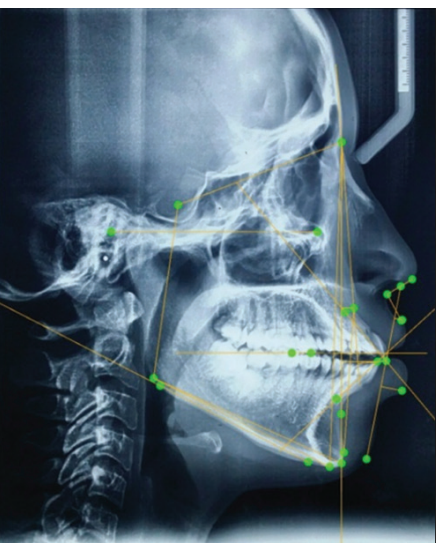

Figure 3: Identification of landmarks and cephalometric analysis

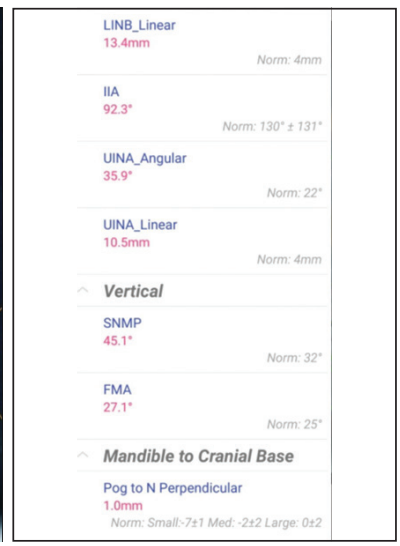

Figure 4: Measurement of various cephalometric parameters 


\section{Statistical analysis}

All the statistical analysis was performed on SPSS software version 22.0. The results were evaluated with the $95 \%$ confidence intervals (Cls), and the significance level was set at $p<$.05.Data were then evaluated with the paired samples $t$ tests. After 10 days, the intra-examiner reliabilities were assessed based on 10 new randomly selected tracings. The reliabilities of the measurements were evaluated by performing Interclass Correlation Coefficient (ICC) analyses with the $95 \% \mathrm{Cls}$.

\section{RESULT}

The mean values, standard deviation and difference in mean between the manual and application tracing method are presented in Table 1.There was significant difference in cephalometric tracing duration between the manual method and application method. Cephalometric tracing duration of manual method was found to be significantly higher $(p<0.05)$ than that of the app method. There was no significant difference in values of SNA, SNB, ANB, Nasion perpendicular to point A, Nasion perpendicular to pogonion(pog), Angle of convexity, Go-GN/SN, FMA, U1-NA (degree), U1-NA(linear), L1-NB (degree), Cant of Occlusion, Interincisal angle, upper lip to Steiner's $S$ line, lower lip to Steiner's $S$ line between the manual and application method (Table 1). The values of linear measurement between Lower incisor to NB line showed significant difference $(\mathrm{p}<0.05)$ (Table 1).The ICC values for intra examiner reliability for all cephalometric measurement with manual and application method ranged between 0.910 and0.998 (Table 2).

Table 1: Cephalometric values obtained from manual and application methods and their differences

\begin{tabular}{|c|c|c|c|c|c|}
\hline SN & Parameters & $\begin{array}{l}\text { Manual Cephalometric } \\
\text { tracing mean value (M), } \\
\text { Standard deviation(SD) }\end{array}$ & $\begin{array}{c}\text { APP cephalometric tracing } \\
\text { mean value, } \\
\text { standard deviation(SD) }\end{array}$ & $\begin{array}{c}\text { Difference between app } \\
\text { tracing and } \\
\text { manual tracing }\end{array}$ & P-value \\
\hline 1. & Performing Duration & $15.588 \pm 0.832$ & $4.852 \pm 0.604$ & $10.735 \pm 0.896$ & $<0.001^{*}$ \\
\hline 2. & SNA & $82.171 \pm 3.584$ & $82.334 \pm 3.648$ & $-0.0162 \pm 1.186$ & 0.432 \\
\hline 3. & SNB & $78.285 \pm 3.678$ & $78.371 \pm 4.046$ & $-0.085 \pm 0.997$ & 0.614 \\
\hline 4. & ANB & $3.985 \pm 2.886$ & $4.060 \pm 2.890$ & $-0.074 \pm 0.084$ & 0.608 \\
\hline 5. & $N$ per to point $A$ & $1.457 \pm 3.313$ & $1.288 \pm 3.644$ & $0.168 \pm 1.655$ & 0.512 \\
\hline 6. & N per to pogonion & $-3.331 \pm 4.742$ & $-3.374 \pm 5.192$ & $0.060 \pm 1.336$ & 0.792 \\
\hline 7. & Angle of convexity & $5.942 \pm 6.700$ & $5.588 \pm 7.224$ & $0.354 \pm 1.859$ & 0.267 \\
\hline 8. & Cant of occlusion & $8.242 \pm 4.308$ & $8.954 \pm 4.121$ & $-0.711 \pm 1.691$ & 0.127 \\
\hline 9. & FMA & $25.542 \pm 6.625$ & $26.234 \pm 6.343$ & $-0.691 \pm 2.417$ & 0.100 \\
\hline 10. & SnGoGn & $31.171 \pm 6.549$ & $31.491 \pm 6.973$ & $-0.320 \pm 1.160$ & 0.112 \\
\hline 11. & Jaraback's ratio & $64.670 \pm 5.795$ & $64.922 \pm 6.032$ & $-0.251 \pm 2.338$ & 0.529 \\
\hline 12. & $\begin{array}{l}\text { Upper incisor to NA } \\
\text { angle }\end{array}$ & $27.542 \pm 9.102$ & $27.585 \pm 9.291$ & $0.428 \pm 1.690$ & 0.882 \\
\hline 13. & $\begin{array}{l}\text { Upper incisor to NA } \\
\text { linear (mm) }\end{array}$ & $6.471 \pm 2.682$ & $6.200 \pm 2.984$ & $0.271 \pm 0.962$ & 0.104 \\
\hline 14. & $\begin{array}{l}\text { Lower incisor to NB } \\
\text { angle }\end{array}$ & $30.000 \pm 10.729$ & $30.037 \pm 10.561$ & $-0.037 \pm 1.153$ & 0.850 \\
\hline 15. & $\begin{array}{l}\text { Lower incisor to NB } \\
\text { linear }(\mathrm{mm})\end{array}$ & $6.228 \pm 4.362$ & $5.715(3.944)$ & $0.511 \pm 0.696$ & $<0.001^{*}$ \\
\hline 16. & Interincisal angle & $119.906 \pm 19.252$ & $118.631 \pm 17.589$ & $1.275 \pm 0.806$ & 0.135 \\
\hline 17. & Nasolabial angle & $100.514 \pm 11.049$ & $100.791 \pm 11.298$ & $-0.277 \pm 1.032$ & 0.122 \\
\hline 18. & Upper lip to $S$ line (mm) & $2.000 \pm 3.084$ & $2.051 \pm 3.007$ & $-0.051 \pm 0.581$ & 0.604 \\
\hline 19. & Lower lip to S line & $2.571 \pm 3.084$ & $2.574 \pm 3.266$ & $-0.002 \pm 0.749$ & 0.982 \\
\hline
\end{tabular}

${ }^{*} p<0.05$ 
Table 2: ICC( Interclass Correlation Coefficient ) values of Manual and Application tracing

\begin{tabular}{|l|l|l|}
\hline \multicolumn{1}{|c|}{ Parameters } & \multicolumn{1}{|c|}{$\begin{array}{c}\text { Manual tracing } \\
\text { ICC (95\% Confidence Interval) }\end{array}$} & $\begin{array}{c}\text { Manual Cephalometric tracing mean } \\
\text { value (M), Standard deviation(SD) } \\
\text { ICC (95\% Confidence Interval) }\end{array}$ \\
\hline SNA & $0.996(0.982-0.999)$ & $0.995(0.981-0.999)$ \\
\hline SNB & $0.991(0.963-0.998)$ & $0.998(0.991-0.990)$ \\
\hline ANB & $0.995(0.980-0.999)$ & $0.995(0.981-0.990)$ \\
\hline N per to point A & $0.977(0.989-0.999)$ & $0.997(0.988-0.999)$ \\
\hline N per to pogonion & $0.970(0.881-0.993)$ & $0.956(0.985-0.999)$ \\
\hline Angle of convexity & $0.998(0.990-0.999)$ & $0.998(0.994-1.000)$ \\
\hline Cant of occlusion & $0.993(0.970-0.998)$ & $0.989(0.956-0.997)$ \\
\hline FMA & $0.910(0.635-0.978)$ & $0.970(0.880-0.993)$ \\
\hline SnGoGn & $0.956(0.821-0.989)$ & $0.991(0.965-0.998)$ \\
\hline Jaraback's ratio & $0.978(0.910-0.994)$ & $0.978(0.912-0.995)$ \\
\hline Upper incisor to NA angle & $0.991(0.962-0.998)$ & $0.995(0.979-0.999)$ \\
\hline Upper incisor to NA linear (mm) & $0.962(0.847-0.991)$ & $0.976(0.916-0.995)$ \\
\hline Lower incisor to NB angle & $0.985(0.939-0.996)$ & $0.995(0.918-0.990)$ \\
\hline Lower incisor to NB linear (mm) & $0.961(0.843-0.990)$ & $0.996(0.985-0.999)$ \\
\hline Interincisal angle & $0.998(0.990-0.999)$ & $0.996(0.985-0.999)$ \\
\hline Nasolabial angle & $0.958(0.830-0.990)$ & $0.988(0.951-0.997)$ \\
\hline Upper lip to S line (mm) & $0.977(0.907-0.994)$ & $0.993(0.970-0.998)$ \\
\hline Lower lip to S line & $0.931(0.724-0.983)$ & $0.954(0.925-0.958)$ \\
\hline
\end{tabular}

\section{DISCUSSION}

Developments in computer technology have lead to increasing use of digital systems both for tracing and analyzing cephalometric films. ${ }^{10}$ Use of smartphone by physicians and dentist is increasing rapidly day-by-day. A smart phone has capabilities of a complete operating system and with the use of mobile applications or "apps", the single-purpose cell phone has become a handheld computer." Numerous smartphone apps that are related to orthodontics have been used by orthodontic clinicians and patients. ${ }^{12}$ Mamillapalli et al developed and used different smartphone apps for model analysis in orthodontics and the determination of cervical vertebra maturation. ${ }^{11,13}$ Aim of the present study was to compare the cephalometric tracing duration and the results of tracing between the manual and app methods for cephalometric analysis. Here we used to OneCeph mobile application on android version.

In the present study, cephalometric tracing duration was significantly lower while using mobile application than that of conventional manual method. Sayar et al. in their study to compare the results of conventional manual cephalometric tracing with those acquired with smartphone application cephalometric tracing also found the similar results. ${ }^{9}$ Our result was also consistent with the study of Chen et al. which showed significantly less clinical time required to perform cephalometric via the use of a computerized tracing method than that with traditional manual tracing with a ruler and aprotractor..$^{14}$ In our study the app method was substantially faster than manual tracing method.

In our study, the majority of cephalometric parameters showed no significant difference between the conventional and app method. Our results were consistent with the findings of the study conducted by Chen et al. and Paixao et al. who found no significant differences in any of measurements between digital cephalometric tracing and manual cephalometric tracing. ${ }^{3,14}$ However, in our study where we used mobile application, their study was done using computerized digital cephalometric measurement. In another study conducted by Gayatri et al. ${ }^{15}$ they found no significant difference in values of cephalometric parameters of steiner's analysis obtained for manual method and mobile application. Our results were also consistent with the findings of their study. In their study they used Cephninja mobile application whereas OneCeph was used in the present study. Polat-Ozsoy $\mathrm{O}$ et al. in their study found that, despite some discrepancies in measured value between hand tracing and the 
computerized method, any differences were minimal and clinically acceptable and majority of parameter showed no difference. ${ }^{16}$ Results of our study was consistent with their findings. In contrast to our study, Sayar et al. found significant difference among majority of cephalometric parameters where they revealed that most of the measurements in app tracing method were found higher than that of the manual tracing method. ${ }^{\text {? }}$

In the present study majority of cephalometric measurement showed no statistically significant difference between manual and mobile application method except lower incisor to NB linear distance which showed statistically significant difference. The statistical significant difference may be due to zoom in function of application method which made in very sensitive during the detection of the landmark of the lower incisors; moreover due to small values of linear measurement, statistically significant difference might have been occurred. In this study, reliability of measurement of cephalometric parameters using both the method was checked using ICC values. ICC value for both the method were greater than 0.9 which indicates excellent reliability. It was reported that ICC values higher than 0.80 defines a strong reliability.

\section{CONCLUSION}

In the present study no statistical significant difference was seen between majority of cephalometric parameters between manual and mobile application method except linear measurement between lower incisors to NB line. Although there was no significant difference in cephalometric measurements, cephalometric tracing duration using Smartphone application was significantly faster than that of conventional method.

\section{OJN}

\section{REFERENCES}

1. Athanasiou AE. Orthodontic cephalometry. London: Mosby-Wolfie; 1995. p. 9.

2. Erkan M, Gurel HG, Nur M, Demirel B. Reliability of four different computerized cephalometric analysis programs. The European Journal of Orthodontics. 2011 Apr 18:34(3):318-21.

3. Paixao MB, Sobral MC, Vogel CJ, et al. Comparative study between manual and digital cephalometric tracing using Dolphin Imaging software with lateral radiographs. Dental Press J Orthod.2010;15:123-130.

4. Leonardi R, Giordano D, Maiorana F, Spampinato C. Automatic cephalometric analysis. Angle Orthod 2008:78(1):145-51.

5. Millward Brown Study, AdReaction. Marketing in a multiscreen world; [Internet]. 2014. Available from: http://www.millwardbrown.com/ AdReaction/2014/\#/

6. Ozdalga E, Ozdalga A, Ahuja N. The smartphone in medicine: a review of current and potential use among physicians and students.J Med Internet Res. 2012; 14:e128.

7. Mohan AT, Branford OA. iGuide to plastic surgery: iPhone apps, the plastic surgeon, and the health care environment. AesthetSurg J. 2012; 32:653-658.

8. Shettigar P, Shetty S, Naik RD, Basavaraddi SM, Patil AK. A Comparative Evaluation of Reliability of an Android-based App and Computerized Cephalometric Tracing Program for Orthodontic Cephalometric Analysis. Biomedical and Pharmacology Journal. 2019 Mar 25;12(1):341-6.

9. Sayar G, Kilinc DD. Manual tracing versus smartphone application (app) tracing: a comparative study. Acta OdontologicaScandinavica. 2017 Nov 17;75(8):588-94.

10. Celik E, Polat-Ozsoy O, ToygarMemikoglu TU. Comparison of cephalometric measurements with digital versus conventional cephalometric analysis. The European Journal of Orthodontics. 2009 Feb 23;31 (3):241-6.

11. Pavan KM, Praveen KN, Murthy S. Model analysis on a smartphone.J Clin Orthod. 2012;46:356-358.

12. Singh P. Orthodontic apps for smartphones. J Orthod. 2013;40:249-255.

13. Mamillapalli PK, Sesham VM, Neela PK, et al. A smartphone app for identifying cervical vertebral maturation stages. J Clin Orthod.2015; 49:582-585.

14. Chen SK, Chen YJ, Yao CC, et al. Enhanced speed and precision of measurement in a computer-assisted digital cephalometricanalysis system. Angle Orthod. 2004;74:501-507

15. Gayatri G, Harsanti A, Zenab Y, Sunaryo IR. Steiner cephalometric analysis discrepancies between conventional and digital methods using Cephninjaß application software. Padjadjaran Journal of Dentistry. 2016 Nov 30;28(3).

16. Polat-Ozsoy O, Gokcelik A, Toygar Memikoglu TU. Differences in cephalometric measurements: a comparison of digital versus handtracing methods. The European Journal of Orthodontics. 2009 Jun 1;31 (3):254-9. 